\title{
Makkah is the Ultimate Multilingual Phenomenon
}

\author{
MUNASIR ALHAMAMI ${ }^{1}$
}

\begin{abstract}
The majority of the published studies on multilingualism concentrate on education, while only a few published papers describe this concept outside the education environment. The current descriptive study uses Makkah City as an example to describe two multilingual phenomena: Umrah and Hajj as examples of permanent and temporary multilingualism phenomena, respectively. This study uses government and published statistics to illustrate each multilingual phenomenon. First, this research describes temporary multilingual phenomenon and uses Hajj to illustrate it. Second, the current study describes permanent multilingualism phenomenon by using Umrah as an example. Third, this study discusses the issues related to the diversity of native speakers in one location. Lastly, research agendas are suggested.
\end{abstract}

Keywords: communication, Hajj, Makkah, multiculturalism, multilingualism

Multilingualism is an evolving phenomenon in different regions of the world. Butler (2013: 111) analyzed the most common definition of "multilingualism" and defines it as "individuals or groups of people who obtain communicative competence in more than one language, with various degrees of proficiencies, in oral and/or written forms, in order to interact with speakers of one or more languages in a given society". The ease of travel and mobility results in the use of different languages in one location, thereby enabling different places to become considerably multilingual. The ease of mobility brings diversity of languages, nationalities, races, foods, clothes, and cultures. Blommaert (2010) and Weber and Horner (2012) stated that in super-diverse places, different accents, language varieties, and registers are used simultaneously. Super-diversity creates a complex of specific semiotic resources, in which languages, accents, dialects, and registers influence the methods of communication in particular settings. Religious gatherings and international events are examples of the super-diversity of languages, accents, dialects, and registers.

The diversity of languages and cultures is reflected in the diversity of the lifestyles of people who live in a multilingual location. Different signs of the multilingualism are found in one location. For example, the more multilingual a location, the more shopping signs are written in different languages and more diverse foods, clothes, and people behavior are offered. In addition, the more diverse a place, the more currency types are used. One of the most super-diverse places in the world is Makkah, Saudi Arabia. Coulmas (2013) explained that only a few researchers have conducted studies on the sociolinguistic dynamics of written language in multilingual cities, such as shop signs and public instructions. Coulmas stressed that multilingualism in the written mode remains a new and relatively unexplained area of sociolinguistics. Hence, research should be conducted to explore the linguistics landscapes in multilingual cities.

${ }^{1}$ Munassir Alhamami, Ph.D., assistant professor at the Faculty of Languages \& Translation, King Khalid University, ASIR - ABHA 61421, Saudi Arabia, email: mhamame@kku.edu.sa. 
Mecca or Makkah is located in the western region of Saudi Arabia. This city is the holiest city in Islam and one of the most crowded places on Earth, particularly in the Grand Mosque (aka AlMasjid al-Haram) area. The Grand Mosque of Makkah (al-Masjid al-Haram), which is as also called the Great Mosque of Mecca, is the largest mosque in the world. Millions of Muslims travel to Makkah annually to visit the Grand Mosque. This large number of visitors from across the globe has placed Makkah as one of the most multilingual and multicultural cities in the world. Spolsky (2003) stressed that the interaction between language and religion as topics relevant to bilingualism or multilingualism has been relatively little explored, Although, he assumed that the growing recognition of the academic legitimacy of the field of language and religion will increase in applied linguistic research, the area of multilingualism in religious places has not been explored enough since that time.

The more diverse a city, the more the need to speak the languages of business providers and government officials. That is, people who live in a multilingual city should speak more than one language to obtain additional job opportunities. This circumstance is the case in Makkah. People who live in Makkah speak different languages in their business stores to interact with visitors from different countries. Their business requires them to speak more than one language and to know how to deal with different customers from various cultures to sell products. The residents of Makkah learn various languages through their daily contact with this city's visitors from different continents. These visitors observe the diversity of nationalities. The Saudi government issues millions of visas annually for visitors of Makkah. Visas are assigned to countries on a quota basis according to the number of Muslims in each country. However, the Saudi government has imposed additional restrictions on the entry of visitors who have previously been to Mecca. This restriction aims to discourage overcrowding while still accommodating Muslims who have yet to visit Makkah and experience the Hajj.

Although many published studies focus on multilingualism, the majority of the multilingual research discuss education. Only a few studies consider the multilingual places outside the academic environments. The majority of the related research concentrate on multilingualism and superdiversities of people owing to immigration and often for educational purposes (e.g., Blommaert, Rampton, \& Spotti 2011; Creese \& Blackledge 2018; De Saint-George \& Weber 2013). This trend may be caused by the difficulty experienced by language researchers to access places outside educational institutions. The connection between multilingualism researchers and non-educational environment is limited, even though different problems in multilingual places outside educational institutions require researchers to analyze and provide solutions. Hence, research on the multilingualism phenomena outside educational institutions (e.g., schools, colleges, universities, and academies) should be conducted.

This descriptive study considers Makkah an excellent example of the diversity of nationalities. This research also describes two events in Makkah, namely the Hajj and Umrah as temporary and permanent multilingual phenomena, respectively. In particular, placing multilingualism into the two general frameworks enable researchers to describe multilingualism and its related issues.

\section{Temporary Multilingual Phenomenon}

We define temporary multilingual phenomenon as the use of more than one language by a group of people in one sitting during a particular period to achieve specific goals, such as performing Hajj. This period may last for a couple of days or several weeks. The sitting may be a physical location or an online environment. The temporary multilingual phenomenon can be repeated within the year or 
annually. Temporary multilingual nonacademic situations can be viewed through several examples. International championships (e.g., FIFA World Cup and Winter and Summer Olympic Games) represent an example of repeated temporary multilingual phenomena. Meanwhile, international conferences, exhibitions, and meetings (e.g., World Business Forum, World Economic Forum Annual Meeting, European Summits, and The Group of Twenty Summit) represent diversity in languages and cultures. Entertainment events that last for days (e.g., Glasgow International Comedy Festival, Vancouver Fashion Week, DC Environmental Film Festival, MaerzMusik, and Cape Town International Jazz Festival) gather people from different backgrounds.

The analysis of temporary multilingual phenomena entails a variety of challenges. However, a few temporary multilingual phenomena may not last for a long time. The limited time adds difficulty for researchers to analyze the phenomena. Moreover, conducting research may need several weeks for data collection, although a few temporary multilingual phenomena may only last for a couple of days. Another issue is the difficulty in contacting the participants if they are eventually needed because as the majority of the people will leave the country or location of the temporary multilingual phenomenon within a short period. The attendees in temporary multilingual phenomenon change rapidly as well. No consistent systems of participation are observed in the phenomenon. For example, in a few world championships, several countries participate in one year but may be unable to attend in the succeeding years. This instance eliminates the language of this particular country. Another critical factor is the feeling of the researcher when analyzing a temporary multilingual phenomenon. A few researchers may not feel the worth to search for temporary multilingual phenomenon given the short duration. This study uses Hajj as an example of temporary multilingual phenomenon to show diversity.

\section{Hajj as a Temporary Multilingual Phenomenon}

The Hajj is one of the largest annual pilgrimages in the world and is known as the fifth pillar of Islam. Hajj is a religious duty that should be accomplished at least once in the lifetime of a Muslim if he can afford to participate physically and financially. The Hajj lasts for one week and involves performing several rituals within that time. The Hajj pilgrims begin arriving in preparation this pilgrimage on the 10th and 11th lunar months. Although the Hajj rituals themselves last only from the 7th to the 12th day of the 12th lunar month (Zulhijjah), pilgrims from outside Saudi Arabia must have Hajj visas if they come any time from the 10th to the 12th month. Pilgrims come from all countries and speak different languages.

On the 11th month of the lunar calendar, thousands of flights arrive at King Abdul-Aziz International Airport bringing pilgrims from various countries. Hence, the airport is notable for its Hajj terminal, which was built to handle pilgrims arriving for the Hajj. Arab News reported that the Hajj terminal in Jeddah can accommodate 175,000 pilgrims at a time: 91,000 at the arrival lounge and 84,000 in the departure lounge. The Hajj and Umrah Complex covers 510,000 square meters. The airport has 26 aircraft parking spaces, 10 skyways, 18 travel gates, and 143 counters for immigration formalities. The Saudi government spends millions of dollars to provide the best services to the pilgrims. In the following sections, the author will discuss statistics to show the diversity of the Hajj. The statistical tables are sourced from the General Authority for Statistics official website. These statistics illustrates the diversity of the Hajj and the estimated number of languages that are spoken by the pilgrims.

Statistics in the last 10 years indicate an average of 2,383,415 Muslims participate in the Hajj annually. The Hajjis (pilgrims) gather in small places to perform Hajj rituals for five to six days. Table 1 illustrates the number of Hajjis annually in the last decade. Note that the number has decreased in 
the last five years because of construction works in the area of the Hajj. However, the number is expected to increase in the coming years. The Saudi 2030 Vision indicates that the number of pilgrims will increase in the coming years.

Table 1: Total Pilgrims in 10 years (2008-2017)

\begin{tabular}{|c|c|}
\hline Number of Hajj Pilgrims & Year \\
\hline $2,408,849$ & $1429(2008)$ \\
\hline $2,313,278$ & $1430(2009)$ \\
\hline $2,789,399$ & $1431(2010)$ \\
\hline $2,927,717$ & $1432(2011)$ \\
\hline $3,161,573$ & $1433(2012)$ \\
\hline $1,980,249$ & $1434(2013)$ \\
\hline $2,085,238$ & $1435(2014)$ \\
\hline $1,952,817$ & $1436(2015)$ \\
\hline $1,862,909$ & $1437(2016)$ \\
\hline $2,352,122$ & $1438(2017)$ \\
\hline Adapted from Websites of the Ministry of Hajj and Umrah and General Authority for Statistics
\end{tabular}

Table 2 illustrates the number of domestic and foreign hajjis in 2017. The total number of domestic hajjis is 600,108, which comprise 35\% and 65\% Saudi and non-Saudi hajjis, respectively. The domestic hajjis are residents of Saudi Arabia during the time of the Hajj. They perform the Hajj but did not apply for a Hajj visa. The total number of foreign Hajjis is 1,752,014. They come from outside Saudi Arabia to perform the Hajj. Accordingly, Hajj visas were issued to them because they are non-Saudi Arabia residents. Thus, the total number of pilgrims is 2,352,122 in 2017.

Table 2: Hajj Statistics 2017

\begin{tabular}{|l|c|}
\hline \multicolumn{1}{|c|}{ Type of Pilgrims } & Number of Pilgrims \\
\hline Domestic Pilgrims: Saudi Pilgrims & 209,415 \\
\hline Domestic Pilgrims: Non-Saudi Pilgrims & 390,693 \\
\hline Total Domestic Pilgrims & 600,108 \\
\hline Foreign Pilgrims & $1,752,014$ \\
\hline Total Of Pilgrims & $2,352,122$ \\
\hline
\end{tabular}

Adapted from the Websites of the Ministry of Hajj \& Umrah and General Authority for Statistics

Table 3 illustrates the nationalities of domestic pilgrims who performed the Hajj in 2017. Egyptians comprised the highest number of domestic Hajjis who live and work in Saudi Arabia, followed by Pakistanis and Indians. The most spoken languages of domestic Hajjis are Arabic, Urdu, Hindi, Bengali, Indonesian, Filipino, English, Hausa, Malay, and Amharic. Although Arabic is one language, it has different dialects. Hence, a Saudi national may have difficulty in understanding Moroccan Arabic. Accordingly, modern standard Arabic is probably the most spoken Arabic dialect by Arabic domestic Hajjis. 
Table 3: Non-Saudis Domestic Pilgrims in 1438 (2017) By Sex and Nationality

\begin{tabular}{|l|r|r|r|r|}
\hline \multicolumn{1}{|c|}{ Country } & Ratio to Total & Total Number & Female & \multicolumn{1}{c|}{ Male } \\
\hline Egypt & $36.9 \%$ & 36,522 & 14,169 & 22,353 \\
\hline Pakistan & $13.8 \%$ & 13,643 & 3,622 & 10,021 \\
\hline India & $10.7 \%$ & 10,581 & 3,066 & 7,515 \\
\hline Yemen & $8.9 \%$ & 8,853 & 2,998 & 5,855 \\
\hline Jordan & $4.9 \%$ & 4,851 & 1,621 & 3,230 \\
\hline Sudan & $4.6 \%$ & 4,583 & 1,475 & 3,108 \\
\hline Bangladesh & $3.8 \%$ & 3,750 & 547 & 3,203 \\
\hline Syria & $2.7 \%$ & 2,648 & 1,041 & 1,607 \\
\hline Indonesia & $2.3 \%$ & 2,324 & 882 & 1,442 \\
\hline Philippines & $1.4 \%$ & 1,433 & 260 & 1,173 \\
\hline UAE & $1.1 \%$ & 1,084 & 157 & 927 \\
\hline Kuwait & $0.8 \%$ & 823 & 292 & 531 \\
\hline Morocco & $0.6 \%$ & 568 & 249 & 319 \\
\hline Tunisia & $0.6 \%$ & 559 & 185 & 374 \\
\hline US & $0.5 \%$ & 470 & 104 & 366 \\
\hline Palestine & $0.5 \%$ & 459 & 188 & 271 \\
\hline Nigeria & $0.4 \%$ & 438 & 160 & 278 \\
\hline Malaysia & $0.4 \%$ & 415 & 233 & 182 \\
\hline Ethiopia & $0.4 \%$ & 372 & 166 & 206 \\
\hline Lebanon & $0.3 \%$ & 346 & 132 & 214 \\
\hline Other countries & $4.3 \%$ & 4,287 & 1,373 & 2,914 \\
\hline Total & $100 \%$ & 99,009 & 32,920 & 66,089 \\
\hline
\end{tabular}

Adapted from the Websites of the Ministry of Hajj and Umrah and General Authority for Statistics

Table 4 compares the number of non-Saudi pilgrims who traveled to Makkah to participate in the Hajj in 2016 and 2017. The number of pilgrims increased in 2017 for each group with different ratios. The data are grouped based on the country regions. The Gulf Cooperation Council (GCC) countries include Bahrain, Kuwait, Oman, Qatar, and the United Arab Emirates. Saudi Arabia is not included in this ratio. Table 4 shows a $30.8 \%$ increase in the number of non-Saudi pilgrims compared with the prior year.

Table 4: Comparison of Non-Saudi International \& Domestic Pilgrims in 2016 \& 2017 by Country Groups

\begin{tabular}{|l|r|r|r|r|}
\hline \multirow{2}{*}{ Country Groups } & \multicolumn{2}{|l|}{ Difference } & \multicolumn{2}{l|}{ Number of pilgrims in } \\
\cline { 2 - 5 } & \multicolumn{1}{|c|}{ Ratio } & Amount & \multicolumn{1}{l|}{2016} & \multicolumn{1}{c|}{2017} \\
\hline GCC countries & $35.3 \%$ & 9,144 & 25,873 & 35,017 \\
\hline Arab countries excluding GCC countries & $25.9 \%$ & 91,092 & 352,280 & 443,372 \\
\hline Asian countries excluding Arab countries & $37.3 \%$ & 292,455 & 783,030 & $1,075,485$ \\
\hline African countries excluding Arab countries & $11.5 \%$ & 19,520 & 169,104 & 188,624 \\
\hline European countries & $28.4 \%$ & 18,916 & 66,552 & 85,468 \\
\hline North and South America countries and Australia & $28.7 \%$ & 5,143 & 17,914 & 23,057 \\
\hline Total & $30.8 \%$ & 436,270 & $1,414,753$ & $1,851,023$ \\
\hline Adapted from the Websites of the Ministry of Hajj and Umrah and General Authority for Statistics
\end{tabular}




\section{Permanent Multilingual Phenomenon}

A permanent multilingual phenomenon is the use of more than one language by a group of people in one sitting throughout the year to complete tasks, such as performing Umrah. The sitting may be a physical location or an online environment. Different examples or locations show permanent multilingual phenomena throughout the year. Business establishments, such as restaurants, markets, and hotels, in tourist or business cities often attract people from different countries.

For example, international airports where flights arrive from different countries throughout the year (e.g., Hartsfield-Jackson Atlanta International Airport, Beijing Capital International Airport, Dubai International Airport, London Heathrow Airport, and Tokyo Haneda Airport) bring people from different countries. Popular tourist attractions are (e.g., Great Wall of China in China, Eiffel Tower in France, Taj Mahal in India, Niagara Falls in Canada, The Pyramids in Egypt, Colosseum in Italy, Petra in Jordan, Machu Picchu in Peru, and Angkor Wat in Cambodia) also places where several languages are used daily. Another location where permanent multilingual phenomena can be found is in international organizations and institutions (e.g., Headquarters of the United Nations, The United Nations Educational, Scientific and Cultural Organization, The United Nations International Children's Fund, the World Organization of the Scout Movement, International Committee of the Red Cross. Council of Europe (CoE), European Union (EU), World Trade Organization (WTO), International Police Organization (Interpol), and African Union). Online games (e.g., AdventureQuest 3D, Asphalt Xtreme, Arena of Valor, Minecraft, and Supercell) gather players around the globe who speak different languages.

Evidently, conducting research on permanent multilingual phenomena is easier than that on temporary multilingual phenomena because researchers will find sufficient time to collect data and conduct surveys or interviews. A system or pattern can be observed easily by the researcher during research. The number of participants in each language will be more consistent than in temporary multilingual phenomenon. In the current study, the author will use Umrah as an example of permanent multilingual phenomenon to show the super-diversity of languages and cultures in Makkah.

\section{Umrah as a Permanent Multilingual Phenomena}

The Umrah is an Islamic pilgrimage to Makkah. Muslims participate in this pilgrimage throughout the year. By contrast, the Hajj has specific days as specified in the Islamic lunar calendar. pPeople from outside Saudi Arabia can obtain an Umrah visa given that this pilgrimage may be performed at any time of the year. Moreover, people who live in Saudi Arabia can visit Makkah and perform Umrah anytime they want. Umrah visits reach their peak during Ramadan, the Islamic fasting month. Muslims prefer to perform Umrah during Ramadan because this month multiplies the reward of a single action based on Islamic beliefs.

The official website of the Ministry of Hajj and Umrah indicates that over 52,832,376 participated in the Umrah in the last 10 years. The ministry registered the number of visas issued for Umrah over the past decade and show an increase from 3,379,765 in 1429 AH (2008) to 6,745,145 in $1438 \mathrm{AH}$ (2017). The number of pilgrims and visitors will increase in the coming years to match the objectives of the Saudi 2030 Vision and in line with the integrated strategy for the development of the Hajj and Umrah systems to help millions of Muslims perform their duties. Egypt has sent the most number of Umrah pilgrims in 2017 at 1.15 million. Pakistan came second with 761,330 and Indonesia came third with 635,990 pilgrims. Table 5 illustrates the number of pilgrims who came from outside Saudi Arabia to do Umrah in the last decade. The Ministry of Hajj and Umrah reported

https://doi.org/10.24035/ijit.14.2018.006 
that approximately 6.75 million visas have been issued for Muslims for Umrah. This number is an increase of nearly $6 \%$ compared with that of the previous year when 6.39 million Umrah visas were issued.

Table 5: Umrah Statistics in the last 10 years

\begin{tabular}{|c|c|}
\hline Year & Number of visas \\
\hline $1429(2008)$ & $3,379,765$ \\
\hline $1430(2009)$ & $3,689,110$ \\
\hline $1431(2010)$ & $3,979,761$ \\
\hline $1432(2011)$ & $5,202,256$ \\
\hline $1433(2012)$ & $5,858,720$ \\
\hline $1434(2013)$ & $5,341,974$ \\
\hline $1435(2014)$ & $6,322,941$ \\
\hline $1436(2015)$ & $5,949,212$ \\
\hline $1437(2016)$ & $6,393,492$ \\
\hline $1438(2017)$ & $6,745,145$ \\
\hline Total & $52,832,376$ \\
\hline
\end{tabular}

Adapted from the Websites of the Ministry of Hajj and Umrah and General Authority for Statistics

Table 6 compares the number of Umrah participants in the last two years based on nationalities. This table shows a decrease in Umrah participants from such countries as Egypt, Turkey, and Jordan. The Ministry of Hajj and Umrah website indicates that the decrease in the number of participants from these countries is due to economic and security conditions. The ministry reports also stated an increase in the number of Umrah pilgrims from the US, Germany, Norway, and other countries. In 2017, Pakistanis topped the list of Umrah pilgrims $(1,446,284)$ with an increase of $45 \%$ from the previous year. Indonesians came second $(875,958$ pilgrims) with $25 \%$ increase from the previous year, followed by India (524,604 pilgrims), Bangladesh (80,977 pilgrims) and Iraq $(264,878$, withan increase of 58\% compared with the previous year).

Table 6: Top countries in the last two years who performed Umrah

\begin{tabular}{|lr|lr|}
\hline & & & $1438(2017)$ \\
\hline Egypt & $1437(2016)$ & & $1,446,284$ \\
\hline Pakistan & 991,337 & Indonesia & 875,958 \\
\hline Indonesia & 699,612 & India & 524,604 \\
\hline Turkey & 437,672 & Iraq & 264,878 \\
\hline Jordan & 434,479 & Bangladesh & 80,977 \\
\hline India & 409,639 & & \\
\hline
\end{tabular}

Adapted from Websites of the Ministry of Hajj and Umrah and General Authority for Statistics

One of the main gates to Makkah is the King Abdulaziz International Airport (KAIA). Millions of pilgrims arrive annually to perform Umrah and Hajj using the KAIA. Over 8,588 fights carried the arriving pilgrims while the departing pilgrims were flown on 9,475 flights. On a daily basis, KAIA receives approximately 16,000 arriving pilgrims and sees off 17,000 . According to the 2030 vision website, as many as 30 million pilgrims are expected to perform Umrah in 2030. According to the reported statistics, KAIA's quota of Umrah pilgrims will increase to 9.18 million in 2018, 10.86 million in $2019,12.74$ million in 2020 , and 15.2 million in 2021 . The report also indicates that KAIA's quota of arriving and departing Umrah pilgrims will reach approximately 20 million in 2025. 
KAIA is expected to receive nearly two million people from various regions of the world who will come to Makkah to perform Umrah during the holy month of Ramadan (tentatively to begin May 16, 2018), while the Umrah season will conclude on July 2018. Thereafter, Umrah visas will no longer be issued because the Hajj will start. However, people who reside in Saudi Arabia can still perform Umrah. Inside KAIA, 14 lounges are provided for the arrival and departure to receive and send off the pilgrims, respectively. However, the new expansion of KAIA is expected to open in 2019. The new KAIA has been designed to accommodate over 80 million passengers annually.

\section{Discussions of the Two Multilingual Phenomena}

The following section will discuss issues related to Hajj and Umrah. Moreover, the discussion will be limited to issues that are raised by the super-diversity of the two events. These issues (e.g., learning languages, health services, modern technology use, transportation, and disaster control) are applicable to the majority of the multilingual locations.

\section{Learning Languages and Multilingual Phenomena}

Government agency employees should start to learn additional foreign languages. The purpose is to serve better by effectively responding to queries they receive from the public, mainly Umrah or Hajj pilgrims. The Ministry of Hajj and Umrah initiated programs to learn foreign languages to provide better services for pilgrims. Officials explained that approximately 100 employees of the Passport Department of KAIA speak 9 different languages. Apart from government employees, traders in Makkah have shown interest to learn various languages. The objectives of enhancing communication and bridging the gap in serving pilgrims having different languages (e.g., Urdu, Turkish, English, French, and Persian) have prompted Saudi officials and other stakeholders to learn these languages. Several educational institutions, such as Taibah University and the Islamic University of Madinah, have been offering foreign language courses for the concerned people involved in pilgrimage operations. Urdu may be the most used language in Hajj operations after Arabic because numerous pilgrims come from South Asian countries. An increased demand from different people, including shoppers, is to learn Bahasa (Indonesian). The Indonesian Consulate General has been conducting language classes for Saudi officials (e.g., officials from the Passport Department) and the merchant community. Approximately 100,000 Indonesians visit Saudi every month for the Umrah pilgrimage.

The need for foreign languages in multilingual locations is crucial. Governments should provide additional language courses and focus on language studies more in a city that is known for its multilingualism. In Saudi Arabia, the demand for speakers of different languages should be addressed. Government employees, such as police officers, firefighters, paramedic service providers, should be multilingual speakers. The government should train employees on how to understand different cultures. For example, Taibah University in Madinah is conducting regular language courses for officials involved in Hajj operations annually. Urdu is a priority language even though English, French, Persian, and Turkish are taught to increase the effectiveness of communication between pilgrims and Saudi officials. Urdu is the most used language in the Hajj operations because the large number of pilgrims come from South Asian countries.

\section{Health Services and Multilingual Phenomena}

Providing health information in different languages to Hajj pilgrims is crucial for governments. Different translators are needed in hospitals and medical centers in multilingual places, such as 
Makkah. Their presence could reduce the spread of infectious diseases because they would understand the ailment of foreign pilgrims. The majority of the multilingual events and locations are attended by numerous people from within and outside the host country, geographic region, or continent. One of the significant public health concerns regarding mass gatherings is the importation or exportation of infectious diseases. Such diseases may spread among pilgrims who come from different countries and to the local population. The gathering of a vast number of people from different countries can compromise the health system of the host countries. Moreover, the threat to global health security posed by infectious diseases may be exported to other countries. Accordingly, this issue demonstrates the importance of planning, global communication, and public health surveillance.

\section{Modern Technologies and Multilingual Phenomena}

Technology can play an important role in facilitating communication and guiding people in multilingual locations. For example, an application should be developed for people who perform the Hajj. Mobile applications should likewise be in different languages. At present, the number of mobile phone users has increased substantially and cellphones have become part of the people's life. Today's mobile phones provide voice call and messaging services and several other services. Researchers should utilize mobile phones by designing applications that serve the multilingual phenomena, particularly with the advancement in mobile dictionary and voice recognition applications. The Vision 2030 official website indicates that the Saudi government will improve visa application procedures to improve the process. The government will integrate e-services (e.g., Hajj Services App, Umrah Services App, Manasikana App, and Hajj Bracelets Reader) into the pilgrims' journey, thereby enriching the religious and cultural experiences. These apps are free to download from Google's Android and Apple's iOS. The Ministry of Hajj and Umrah has developed a new mobile application to provide automatic replies to queries on Hajj and Umrah pilgrims through social networking sites. The ministry will eventually launch the app. The app conducts a conversation via auditory or textual methods and works on social networking platforms. The app has basic information required by pilgrims, thereby assisting pilgrims to obtain additional information on the Hajj and Umrah service companies within Saudi Arabia.

\section{Transportation and Multilingual Phenomena}

One of the most challenging issues caused by multilingualism is the difficulty of providing better transportation services. Mobility and transit with people coming from different countries increase transportation problems. The number of Umrah visitors from other countries has tripled, reaching eight million people. The Saudi officials considering to serve pilgrims is a noble responsibility. The Saudi government has likewise begun the third expansion to the Grand Mosque in Makkah, as well as modernizing and increasing the capacities of KAIA. In addition, the government has launched the Makkah Metro project to serve visitors to the Holy Mosques and holy sites in Makkah during Hajj and Umrah. The advancement in the transport system will facilitate access and ASSIST pilgrims perform their visits with greater ease and convenience.

\section{Disaster Control and Multilingual Phenomena}

One of the most challenging factors is controlling disasters and such challenges increase in multilingual locations. People come with different backgrounds and lack familiarity with different government systems. Laws and regulations are different from one country to another. Moreover, 
such natural phenomena as earthquakes, cyclones, volcanic eruption, tsunamis, wildfires, floods, landslides, droughts, and accidents may occur. The deaths and causalities will be higher in multilingual places because people will not react and follow instructions in the same manner. Governments and specialists should have specialized training on how to act in a multilingual environment. General training on how to control disasters will be insufficient because a multilingual environment has unique characteristics that require specialized training. The Hajj has witnessed three common disasters in the past years, namely, fire, suffocation, and stampede. In 1975, approximately 200 pilgrims died as a result of a gas cylinder that exploded. In 1997, approximately 350 died and another 1500 were injured in tent fires. At present, the tens are fireproof. Cases of crushes and failures of crowd control have caused a stampede or a progressive troupe collapse. Other notable incidents that caused the death of numerous pilgrims happened in 1994, 2004, 2006, and 2015.

\section{Instructions, Signs, and Multilingual Phenomena}

The more diverse the location, the more the need to include multilingual signs and instructions in different languages. At present, specialized mobile apps should be used to provide instructions and maps in a multilingual location, such as the Grand Mosque (AlMasjid Alharam). The apps should have all the commonly spoken languages and be free of charge to encourage people to use it. This app may include a map, bathroom areas, food area, transportation, and medical center locations, among others. Such apps will eliminate the related problems and smoothen the transportation process, as well as make people perform their tasks easily. People should also watch educational programs and videos on the multilingual city before they visit. For example, pilgrims should watch educational programs on Makkah and videos that explain the instructions related to Hajj and Umrah before they arrive in Saudi Arabia. Such viewing is necessary for pilgrims who may be unable to use technologies to find their locations if they get lost.

Different methods are available to communicate and understand instructions in Hajj and Umrah, such as body language, facial expressions, reading signs, and asking for help from friends who speak other languages. Moreover, such methods can locate someone who speaks their language, seek assistance from the police where they will connected with translators for all most languages, imitate people doing what they see others do, call friends by using cellphones, or using cellphones to find instruction or information. Other functions could include relaying previous experience on what pilgrams did during their previous visit, remembering what their friends who already visited Makkah said, and recalling what they saw from instructional materials, such as TV shows, videos, or books. Apps include identifying signs, such as common bathroom signs and finding restaurants by looking at what inside the store to buy. Users can understand that a place is selling food by looking at the signs or smelling food. Pilgrims can seek someone who look like they are from their home countries among the Hajj and Umrah performers, as well as people who work in Makkah. Many nationalities are hired by the Saudi government to work in the Masjid to clean prayer areas, as well as electricians and plumbers to fix problems. Pilgrims can understand if these workers are from their countries by asking them what they think as they look like them.

\section{Conferences, Workshops, and Multilingual Phenomena}

The conduct regular conferences, symposiums, seminars, and workshops that discuss the characteristics of specific multilingual location is extremely important. In these conferences, people come from different specializations, such as health, security, transportation, and communication. They gather to discuss updated issues in the multilingual environment. These conferences discuss 
related issues that might arise in the specific multilingual environment due to the diversity of the people and their backgrounds. For the Hajj and Umrah, research and visit conferences are also held. The conference is organized by the Custodian of the Two Holy Mosques' Institute for the Haj and Umrah research in Umm Al-Qura University. The conference also shows the care extended by the Saudi leadership to the pilgrims and visitors. In this annual conference, several researchers, specialists, government officials, and the private sector discuss improvement of services being offered to the pilgrims. Committees (e.g., higher, media, executive, and logistics committees) are formed by the government to organize the international Haj seminar. The seminar will be held at the beginning of the annual pilgrimage.

The study describes two religious events in Makkah to illustrate the super-diversity of people who attend these events. This super-diversity involves different challenges in various areas, such as health, transportation, communication, and disaster control. The two phenomena emerged when diverse groups come together in one location to complete tasks (i.e., temporary and permanent multilingual phenomena). Each phenomenon has its own research challenges and unique characteristics. Although these types of phenomena are depicted in different regions of the world, only a few studies have investigated them. The majority of these studies concentrated on education and immigration problems.

The purpose of this descriptive research is to draw the attention of researchers to investigate multilingualism outside the education environment. Multilingualism can be analyzed in religious places, tourist locations, business shows, and sports events. Several research topics have yet to be explored completely, such as the Hajj, which is the most super diverse multilingual phenomena. When the majority of the world's major spoken languages are used in one city for approximately one week, Makkah becomes one of the most crowded places on Earth. The Saudi government assigns an entire ministry (i.e., Ministry of Hajj) for this event, given that the Hajj is one of the most religiously diverse gatherings on earth.

Different research topics are included in the temporary and permanent multilingual phenomena. The more diverse a place, the more research issues should be analyzed. One of the most important topics is the communication problem. Hence, the following related questions should be answered. How do people communicate in multilingual locations? What strategies do people use to bridge the cultural gaps in multilingual and multicultural locations? How are people's identities preserved or reshaped in super-diverse locations? How do major languages and cultures interact with minor languages and cultures in multilingual and multicultural locations? How do people perceive multilingualism in super-diverse places? How is translanguaging used in multilingualism locations? These proposed research questions will focus on linguistics landscape, translanguaging, perceptions, codeswitching, communication problems and strategies, identity perception, language interactions, and cultural integration.

\section{References}

Blommaert, J. 2010. The Sociolinguistics of Globalization. Cambridge: Cambridge University Press.

Blommaert, J., Rampton, B. \& Spotti, J. 2011. Language and super diversities. Diversity Journal. 13(2): $1-21$.

Butler, Y.G. 2013. Bilingualism/Multilingualism and Second-Language Acquisition. In, T.K. Bhatia \& W.C. Ritchie (Eds.). The handbook of bilingualism and Multilingualism. 2nd ed. pp. 109-136. Oxford, UK: John Wiley \& Sons.

Coulmas, F. 2013. Sociolinguistics: The Study of Speakers' Choices. Cambridge: Cambridge University Press. 
Creese, A. \& Blackledge, A. (eds.) 2018. The Routledge Handbook of Language and Super-Diversity. London: Routledge.

De Saint-George, I., \& Weber, J. 2013. Multilingualism and Multimodality: Current Challenges for Educational Studies. The Netherlands: Sense Publisher.

Hajj (Pilgrimage) Statistics Map - 1438 (2018). General Authority for Statistics: Kingdom of Saudi Arabia. Retrieved from https://hajmap.stats.gov.sa/hajmap/indexmapeng1438.asp.

Hajj Statistics. 2018. General Authority for statistics: Kingdom of Saudi Arabia. Retrieved from https://www.stats.gov.sa/en/page/93.

Spolsky, B. 2003. Religion as a site of language contact. Annual Review of Applied Linguistics. 23: 8194.

Statistics \& Figures of Pilgrims. 2018. Ministry of Hajj and Umrah Website. Retrieved from http://www.haj.gov.sa/english/Pages/default.aspx.

Weber, J. J., \& Horner, K. 2012. Introducing multilingualism: A Social Approach. New York: Routledge. 